\title{
INFLUENCE OF MAV CHARACTERISTICS ON THEIR APPLICATIONS
}

\author{
C. Galinski \\ Warsaw University of Technology, Poland E-mail: cegal@meil.pw.edu.pl \\ Received 1210 2005, accepted 05122005
}

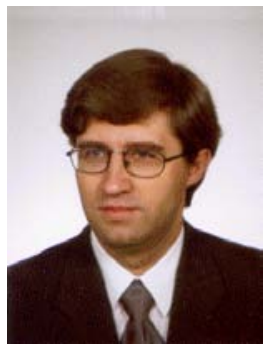

Cezary GALINSKI, $\mathrm{PhD}$

Education: M.Sc (Eng.) 1992, Ph.D. 1997.

Affiliation(s) and function(s): Warsaw University of Technology - Assistant Professor (2004); Cranfield University RMCS - Scientific Fellow and Consultant (2002 - 2004); Research and Development Centre of Mechanical Appliances - Senior Research Engineer (1999 - 2004); Boeing Commercial Airplanes Group - Intern (1998); Warsaw University of Technology - Ph.D. student (1992 - 1997).

Experience: Active fixed and flapping wing MAV researcher since 1999; Participant of NATO Advanced Scientific Fellowship Programme - fixed and flapping wing MAV research; Participant of Boeing post Ph.D. Internship Programme - airplane stability and control research and flight testing; Ph.D. thesis concerning flight testing of UAV's and dynamical similar models; President of SAE Student Chapter at Warsaw University of Technology participant of SAE Aero Design Competition.

Present position: Assistant Professor in the Department of Aircraft and Helicopters at the Warsaw University of Technology

\begin{abstract}
Micro Air Vehicles (MAVs) are an emerging class of unmanned flying vehicles envisaged for direct reconnaissance easy in handling and transport even by single operator. Teams all over the world are developing several different configurations. Each of the configurations exhibits a different set of characteristics. On the other hand, MAVs are expected to serve many different applications and fulfil requirements that sometimes exclude each other. This paper presents the most important characteristics and constraints of fixed wing airplanes, rotary aircraft, and entomopters and show how they fit certain requirements.
\end{abstract}

Keywords: Micro Air Vehicles, characteristics, applications.

\section{Introduction}

A Micro Aerial Vehicle (MAV) is defined here as a flying vehicle as small as practical to perform direct reconnaissance missions, safe, and portable by a single person, including entire ground station. It is envisaged that MAVs will to provide direct reconnaissance in various environments. These environments impose various requirements on the vehicle. On the other hand, there are different concepts of MAV design exhibiting different characteristics. This paper attempts to match these characteristics to the requirements. It is organised as follows. The most important characteristics of fixed wing, rotary, and flapping wing MAVs are described in section 1. Particular attention is paid to flapping wing MAVs since they are the least developed and their characteristics are not widely known. Different missions types are assigned to each MAV type in section 2. Finally, several applications are proposed for each mission and MAV type.

\section{Characteristics of MAVs 1.1 Fixed wing MAVs}

This is the best-developed type of MAV. Several prototypes have been built and proposed to customers already. They exhibit quite good forward flight capabilities: gliding ratio about of eight, maximum speed approximately of $20 \mathrm{~m} / \mathrm{s}$, and flight duration about of one hour. They have power loading around of $13 \mathrm{~kg} / \mathrm{kW}$ for steady, level flight. Fig 1 shows the power required for flight for an MAV built in the delta wing configuration with a weight of $0,25 \mathrm{~kg}$ and wingspan of $0.45 \mathrm{~m}$.

The most important problem encountered so far is the quality of images taken from onboard television equipment. The problem is caused by the necessity to fly in the Earth boundary layer that is turbulent [11]. Turbulence in Earth boundary layer is particularly difficult for MAVs because of their small sizes. This problem can be solved by the use of an advanced control system combined with a highly manoeuvrable aerodynamic configuration [4]. Fig 2 shows one of the possible designs.

The non-standard propeller location helps to keep flow attached to control surfaces even at quite high angles of attack, providing controllability even in strong gusts. Figures 3 and 4 show the effect of the propeller on the flow and resulting lift coefficient.

This effect can probably be explained by unsteady effects similar to those described in $[15,2,6,7]$ but created by the propeller passing through the slot. Similar results can probably be obtained by application of flapping propulsion for fixed wing aircraft [5].

\subsection{Rotary MAVs}

Rotary MAVs have also been quite successful recently. A few designs have reached the flight test phase. Unfortunately, similar to their larger originals, they are 
not particularly efficient. The most advanced design presented by SEIKO EPSON is capable of flying controllably with a payload for only three minutes (Fig 5) $[13,14,12]$. So even if its efficiency was doubled and propulsion efficiency tripled, it would not fly longer than about 20 minutes. On the other hand rotary MAV configurations are the only configurations capable of combining acceptable high and low speed characteristics including hovering.

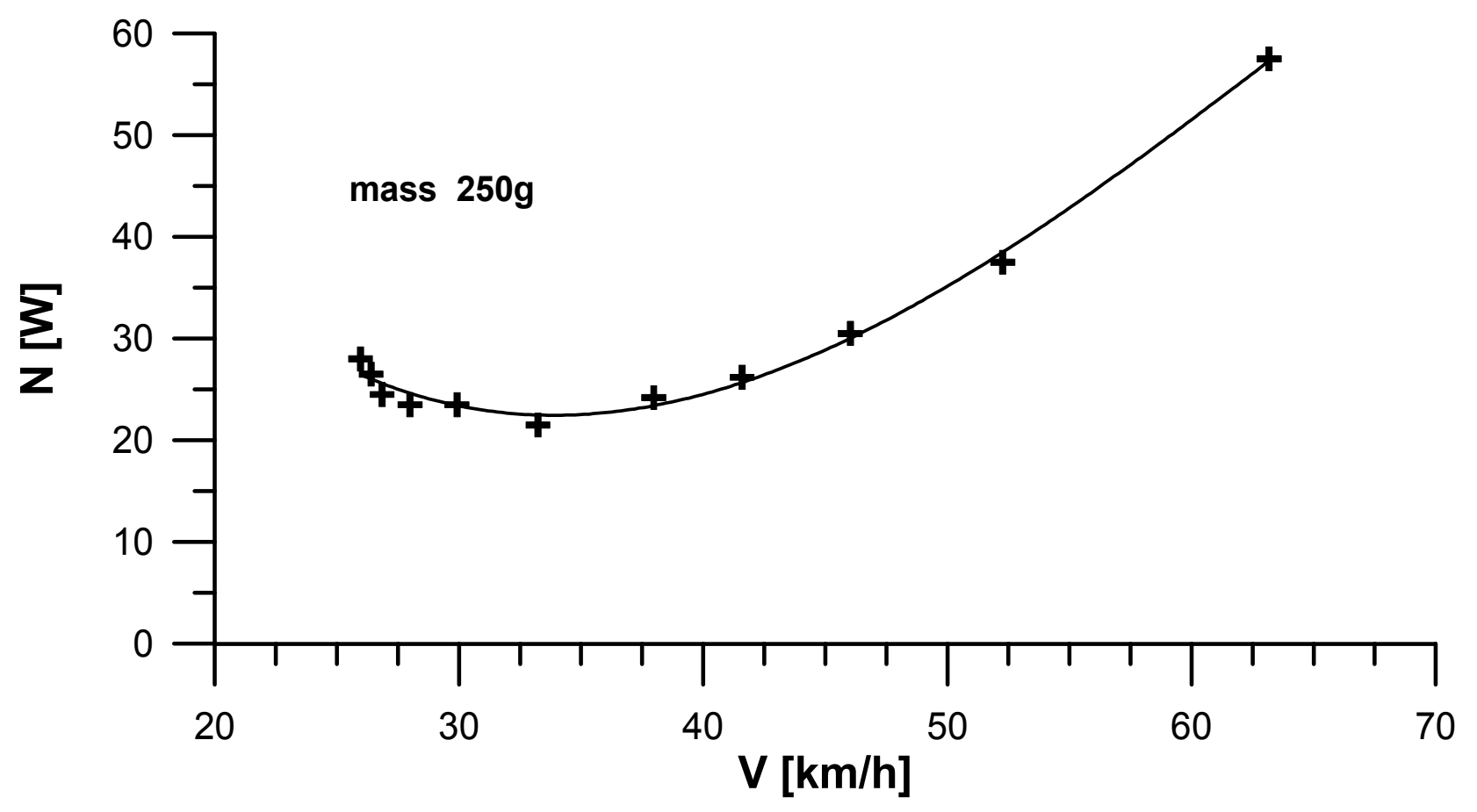

Fig 1. Power required for flight of $250 \mathrm{~g}$ fixed wing MAV

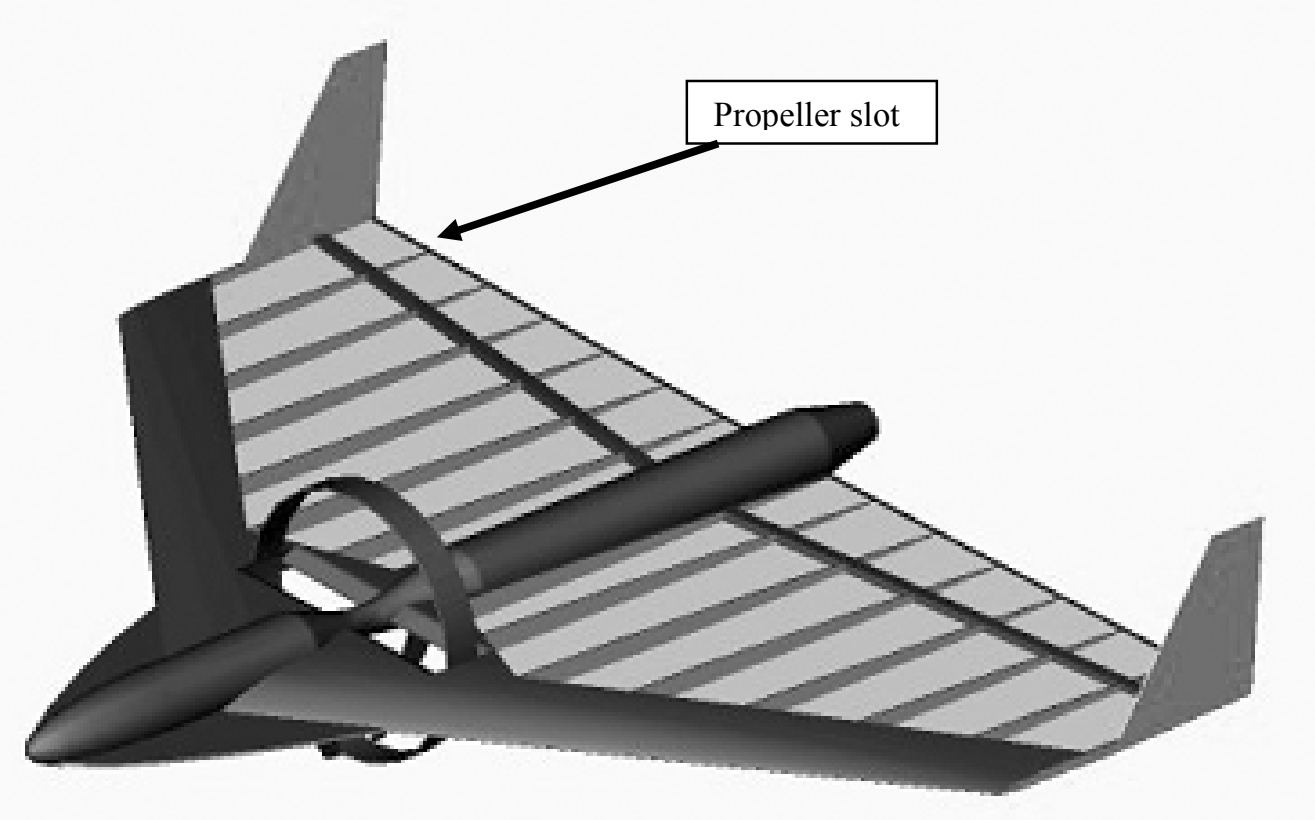

Fig 2. Possible design of gust resistant fixed wing MAV. Note the slot in the wing, where the propeller is to be installed 


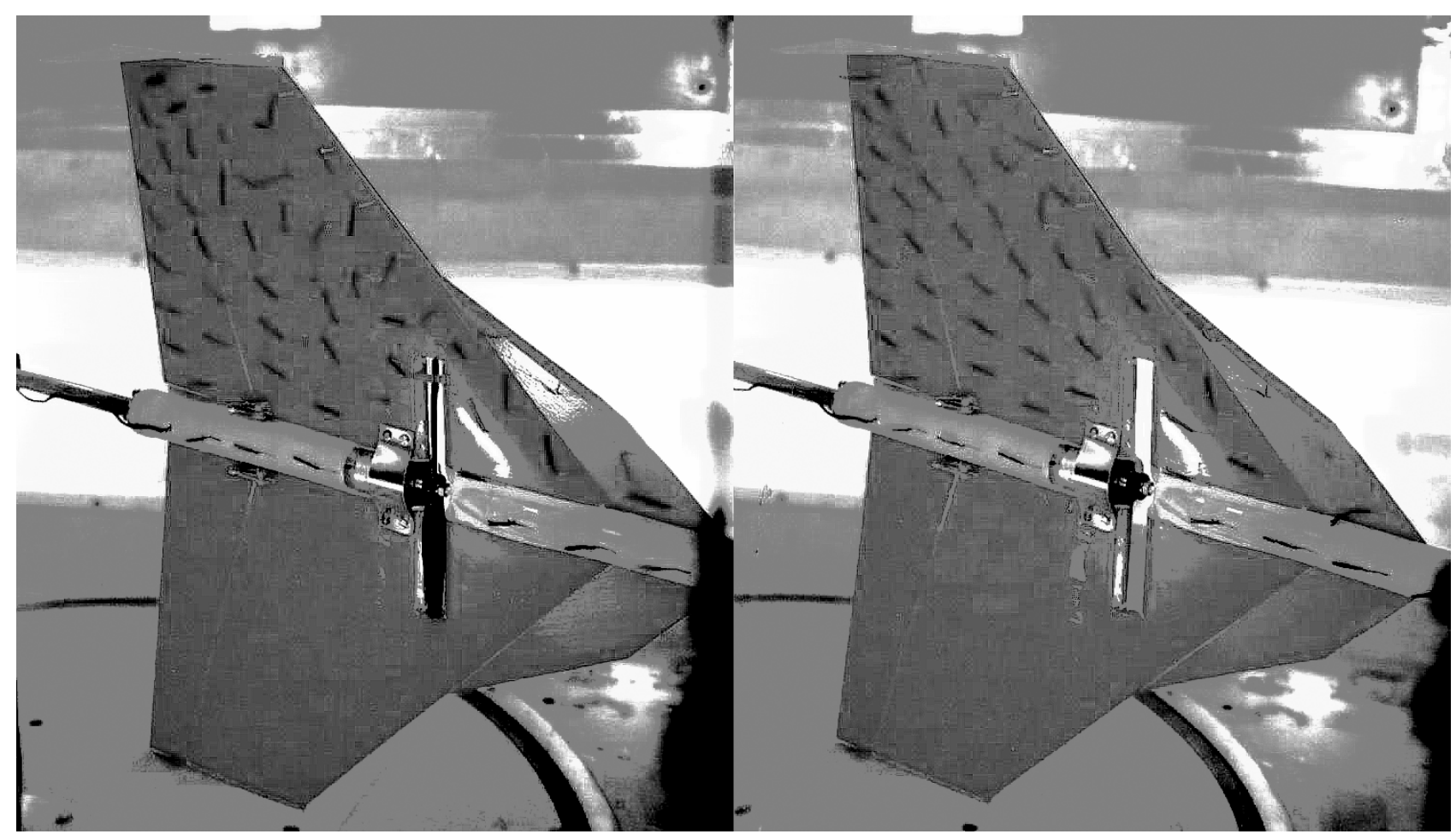

Fig 3. Comparison of flow over the MAV with and without active propulsion for $A o A=20^{\circ}$. A quite well organised flow can be observed when propulsion is active (b), where as large regions of separation are present when the propeller does not rotate (a). This is true not only in the propeller stream, but also close to the wing tip. Of particular interest are the three outboard tufts close to the leading edge. They indicate no flow at all without propulsion and organised flow with propulsion
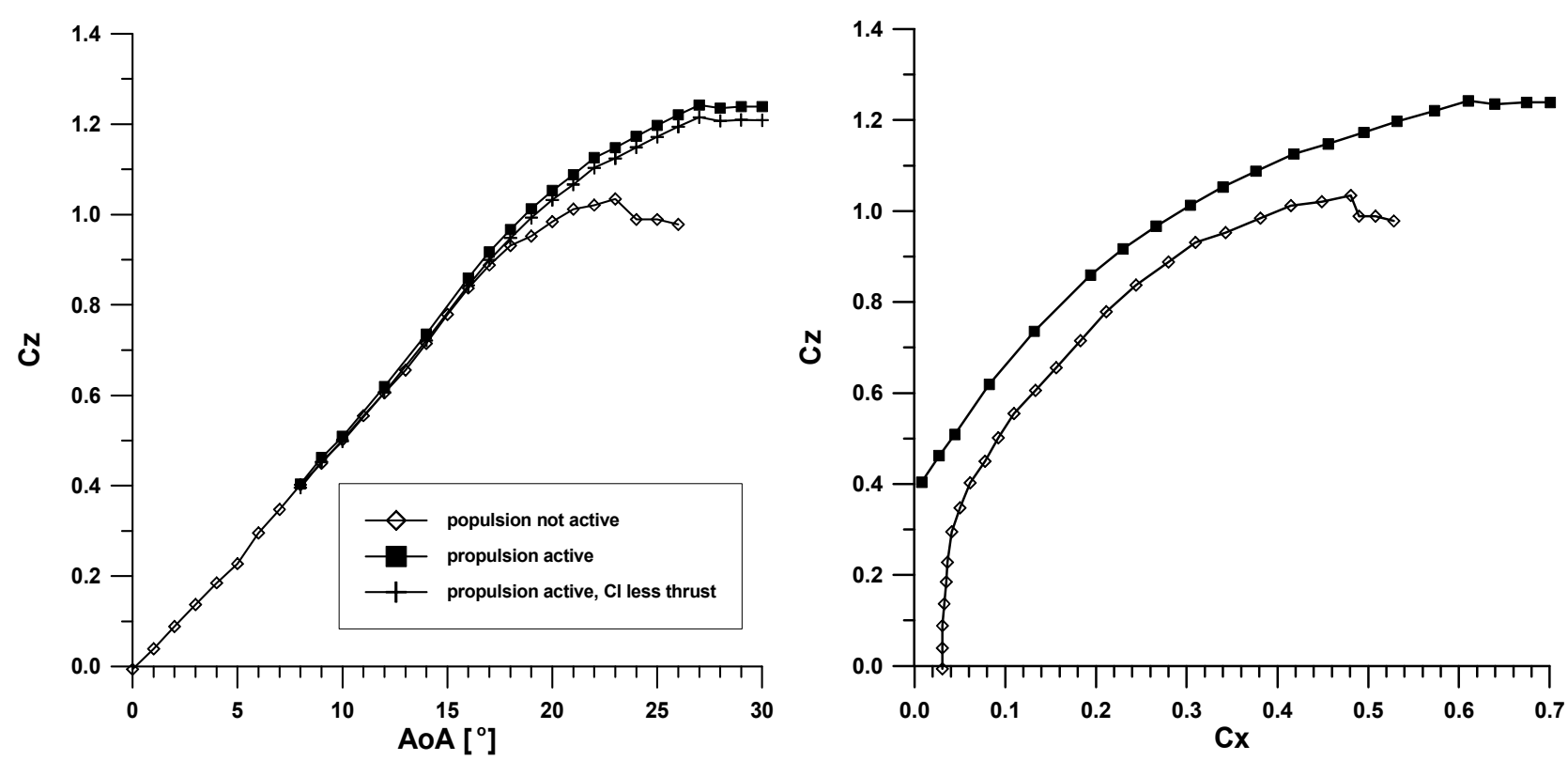

Fig 4. Lift coefficients of the MAV with and without active propulsion. The propeller slot was sealed in the case of measurement without active propulsion

\subsection{Flapping wing MAVs}

This is the least developed group of MAVs. Only a few flying ornithopters have been built build. Flapping wing advantages unfortunately do not occur in the ornithopter mode. Fixed wing airplanes are still more efficient in fast forward flight. On the other hand insects exhibit much greater manoeuvrability and efficiency in hover. Therefore it seems reasonable to attempt the entomopter configuration.

Ornithopters imitate the kinematics of birds, whereas entomopters imitate the kinematics of insects. The major difference between them lies in the variation of the angle of wing setting. Ornithopters generally flap up and down with only minor variation of the angle of wing setting (a 
few degrees), maintaining quite small angles of attack. This allows some thrust to be generated but not enough to hover. They therefore need some horizontal airspeed to take off and rely on gliding ratio when cruising.

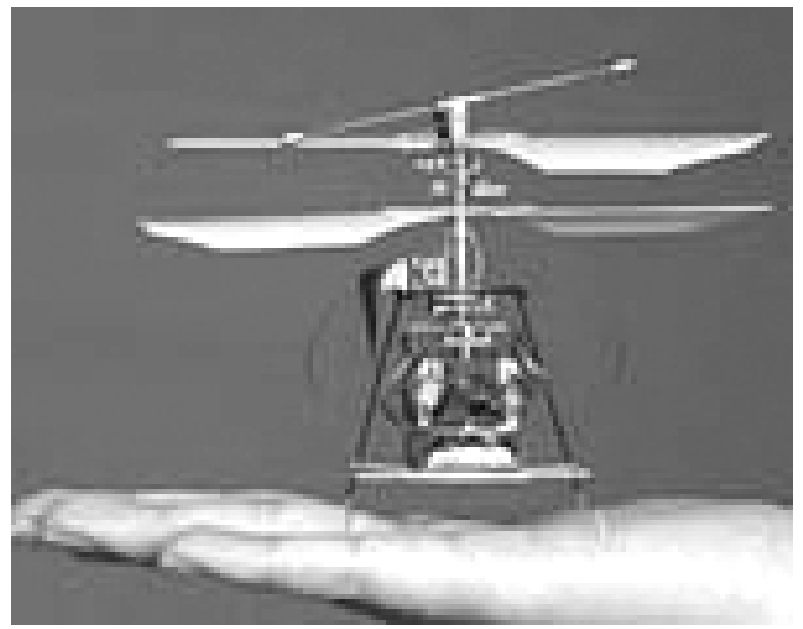

Fig 5. Seiko Epson rotary MAV [8-10]

Entomopters' kinematics assumes large and rapid change in wing angle of wing setting (about 100 degrees) at the end of each stroke. It is therefore often called a "pitch reversal" since the wing is almost flipped over at the end of a stroke (Fig 7). This allows much stronger vortical system to be generated in the flow, including leading edge vortex since high angles of attack are typically applied. As a result, much more thrust is generated. It is believed that this amount of thrust will allow hovering and take off vertically like in the case of insects. Flow complexity unfortunately also creates difficulties in the mathematical description. As a result, only flapping test rigs have been built for aerodynamic experiments so far. The only "flying" prototype is not controllable and too small to carry a payload since it weights only $1 \mathrm{~g}$ [3]. Designs suitable for future application with wingspan of about $0.2 \mathrm{~m}$ and flapping mechanism in the $0.025 \times 0.025 \times 0.025 \mathrm{~m}$ box are just emerging Fig $6[16,17]$.

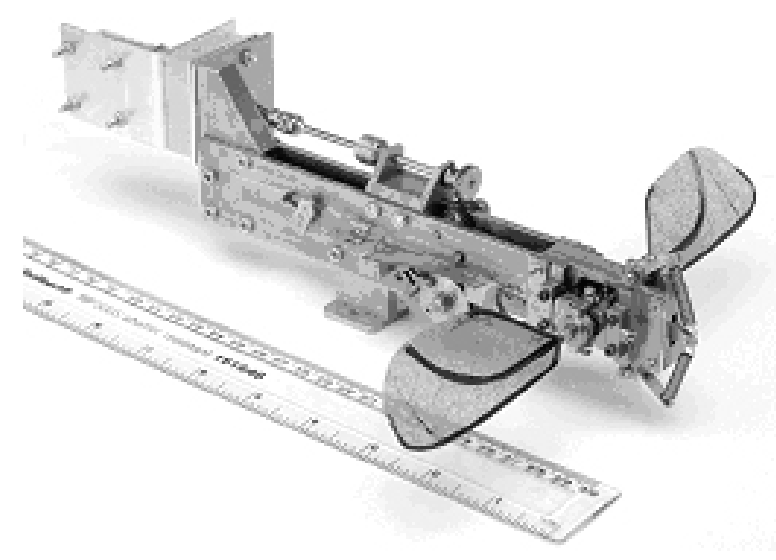

Fig 6. Flapping test rig in the size of future MAV
There are also three large-scale mechanisms designed for aerodynamic testing rather than real flight $[9,10,1]$. They allow time histories of the lift during flapping to be measured. One of them is presented on the Fig 7 according to [8]. Note that advanced pitch reversal means that pitch reversal occurs before the end of the wing stroke end and delayed pitch reversal means that pitch reversal occurs after the end of the wing stroke.

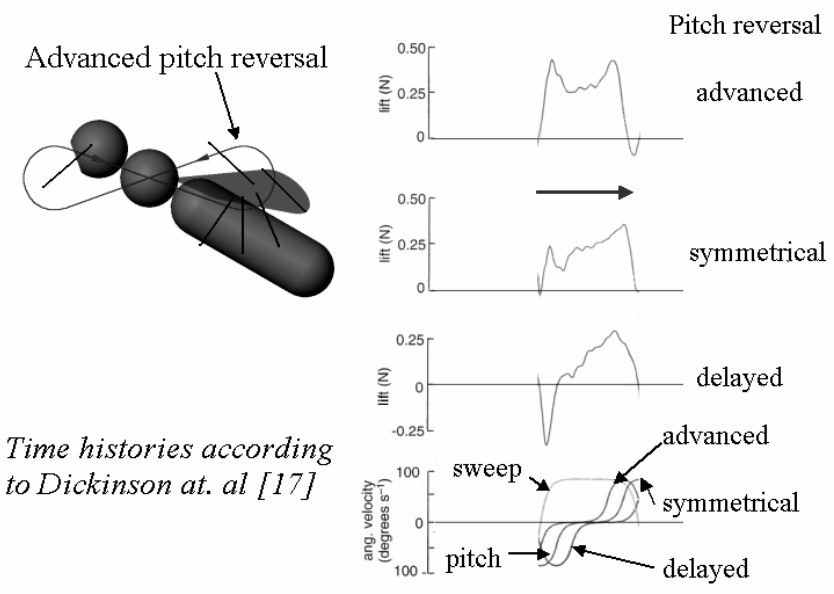

Fig 7. Lift generated by flapping wings

As can be seen from Fig 7, the lift is not uniform during the stroke. There are two lift peaks at the beginning and at the end of each stroke. These lift peaks are still not well described by mathematical formulae, making it difficult to predict MAV performance $[8,18]$. However, it is worth noting that both lift peaks have the same sense as the product $\overrightarrow{\boldsymbol{\omega}} \times \overrightarrow{\mathbf{V}}_{\mathbf{w}}$. Both are directed simultaneously upwards or downwards, never in opposite directions (Fig 8).

It is therefore possible to assume that lift peaks are somehow proportional to this product. This observation allows transient state of the entomopter between hovering and forward flight to be analysed. Let us assume that because of control command pitch, reversal becomes advanced on one stroke end and delayed on the opposite. This will generate the opposite lift peaks on both stroke ends and the resulting global pitching moment in the direction of negative lift peak (delayed wing pitch reversal) (Fig 8).

As a result, forward thrust, forward speed, and drag will be generated (Fig 9). Forward speed however generates its own product $\vec{\omega} \times \overrightarrow{\mathbf{V}}_{\mathbf{0}}$ with the opposite sense to the previous one. The opposite global pitching moment is therefore generated as well. A new state of balance is finally established when both moments become equal (Fig 9).

This mechanism not only explains the method of change between hovering and forward flight, but also shows the main constraint of the entomopter. Pitch reversal advance and delay cannot be increased indefinitely. Fig 10 shows the most advanced and the most delayed reversal of pitch. The most advanced pitch reversal occurs if pitch reversal ends at the same time as 
stroke end. The most delayed pitch reversal occurs if pitch reversal begins at the stroke end (next stroke start). Further advancing and delaying will not increase the speed since lift peaks will occur on shorter arms, thus decreasing the pitching moment generated.
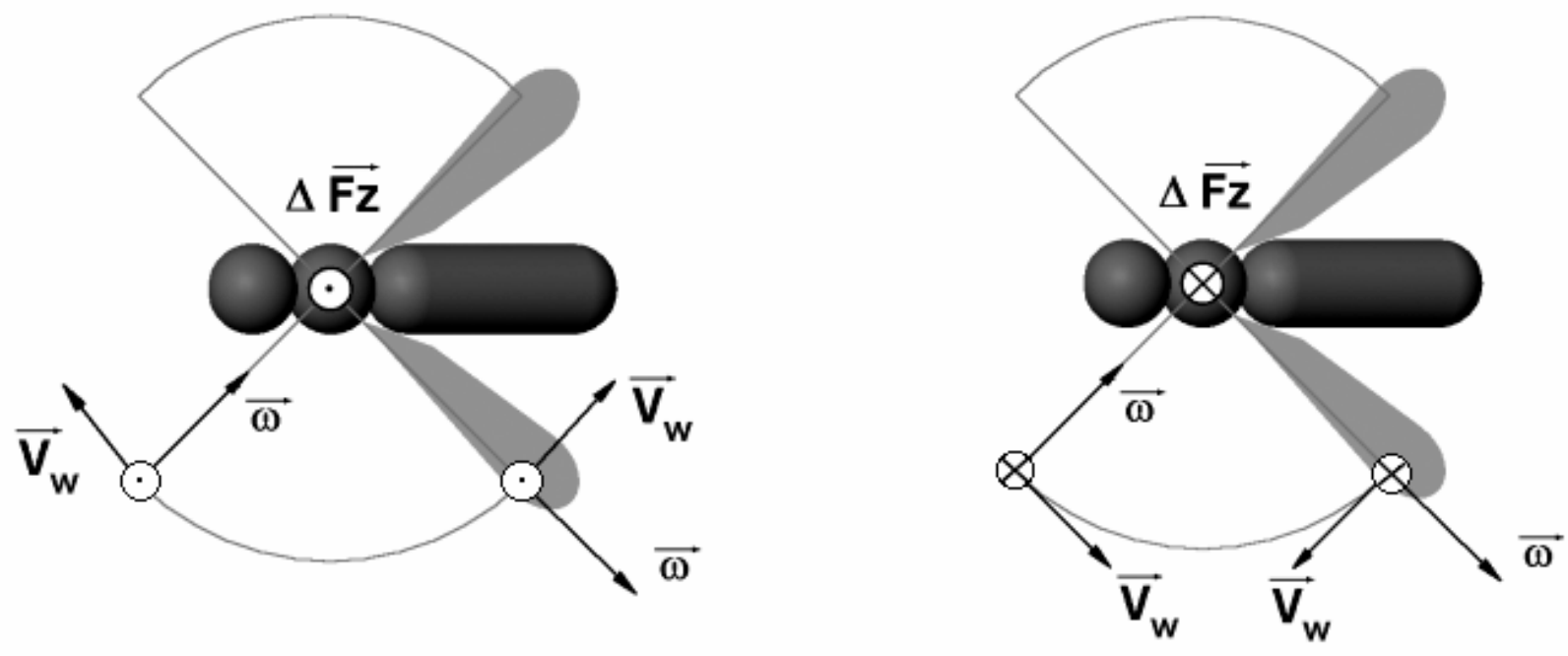

Fig 8. Lift peaks senses

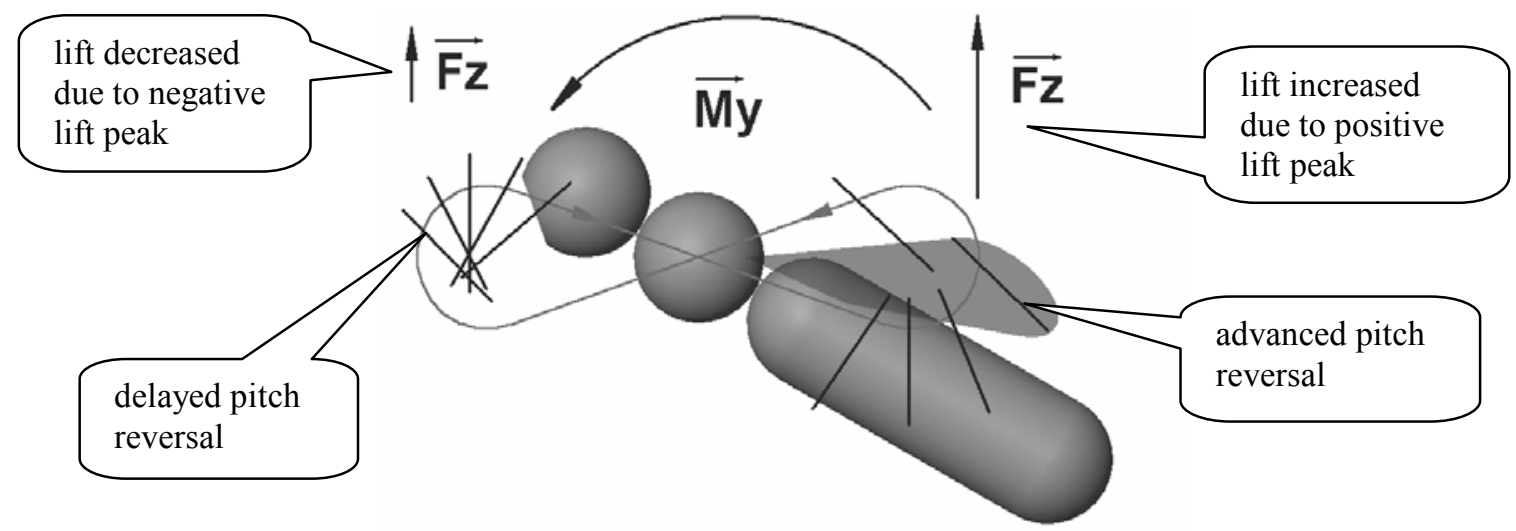

Fig 8. Global pitching moment generated by different wing pitch reversals on both stroke ends
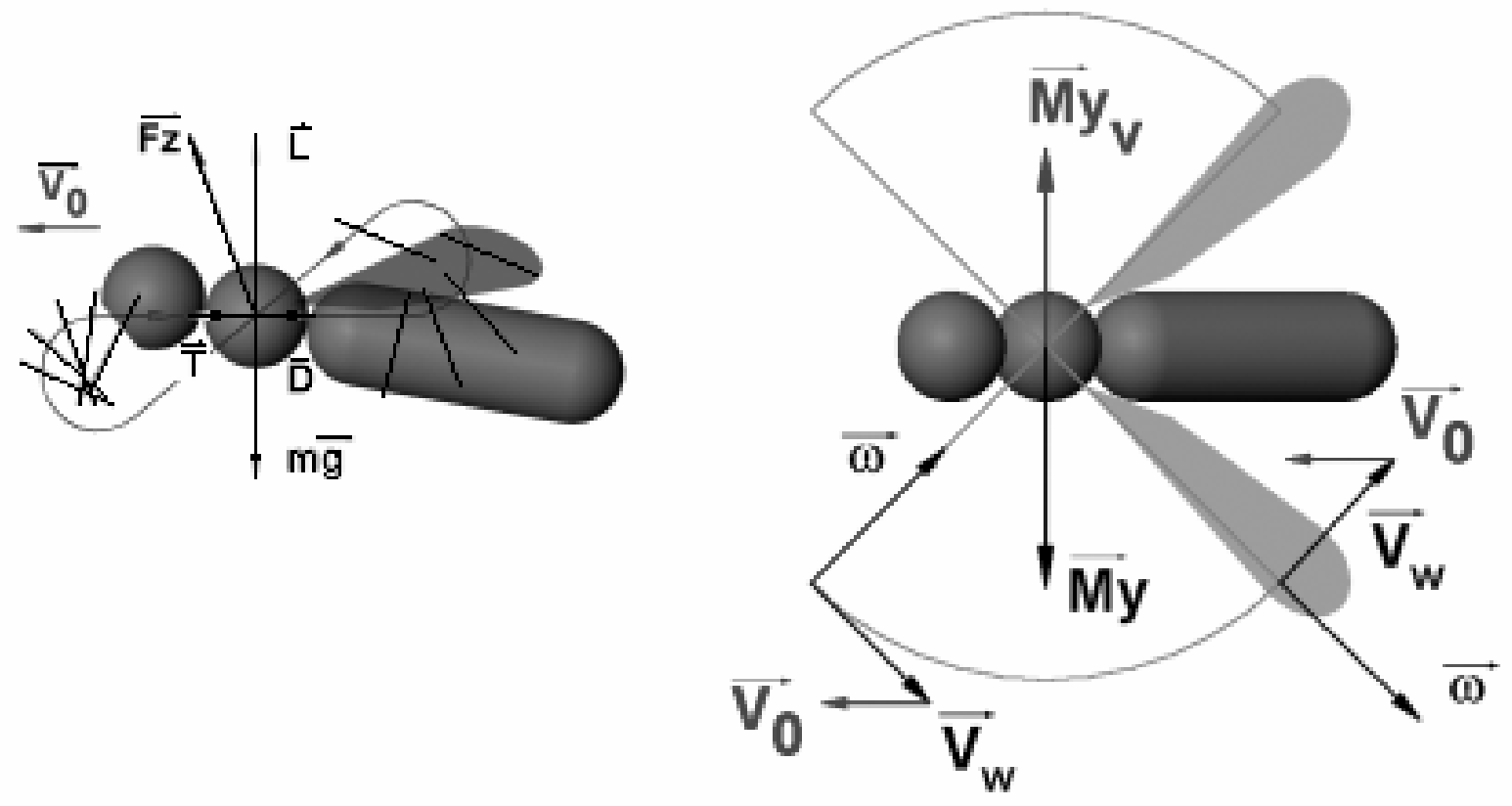

Fig 9. Forces and moments balance for the forward flying flapping MAV 


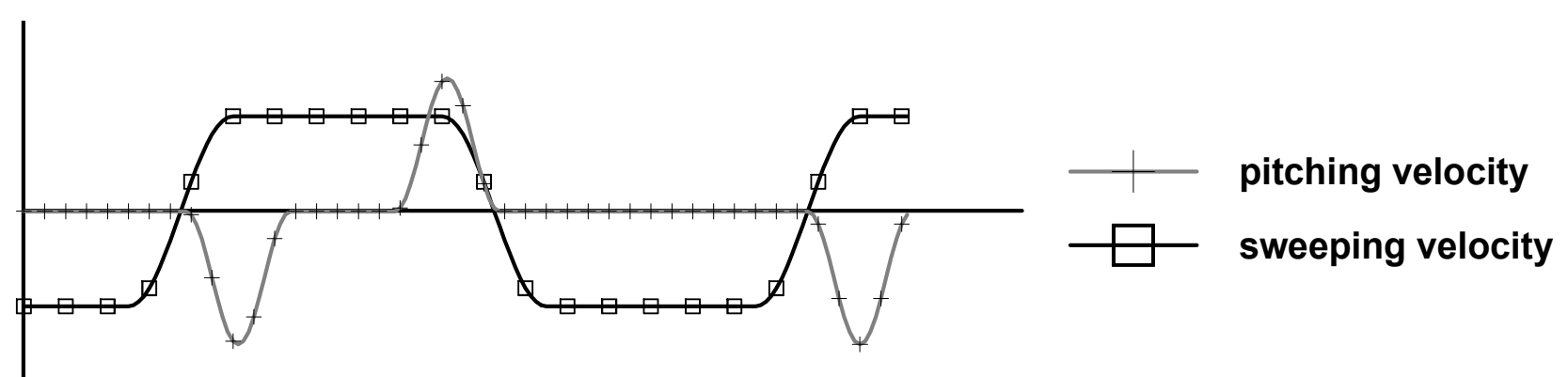

Fig 10. The most advanced and the most delayed pitch reversal

There are also two other methods to increase forward speed. Both are strictly constrained, however.

First of all, it is possible to increase wing linear and/or rotational velocity during pitch reversal. This should increase the value of the lift peak, but also wing loading. Fig 11 shows how the wing loading changes with the flapping frequency.

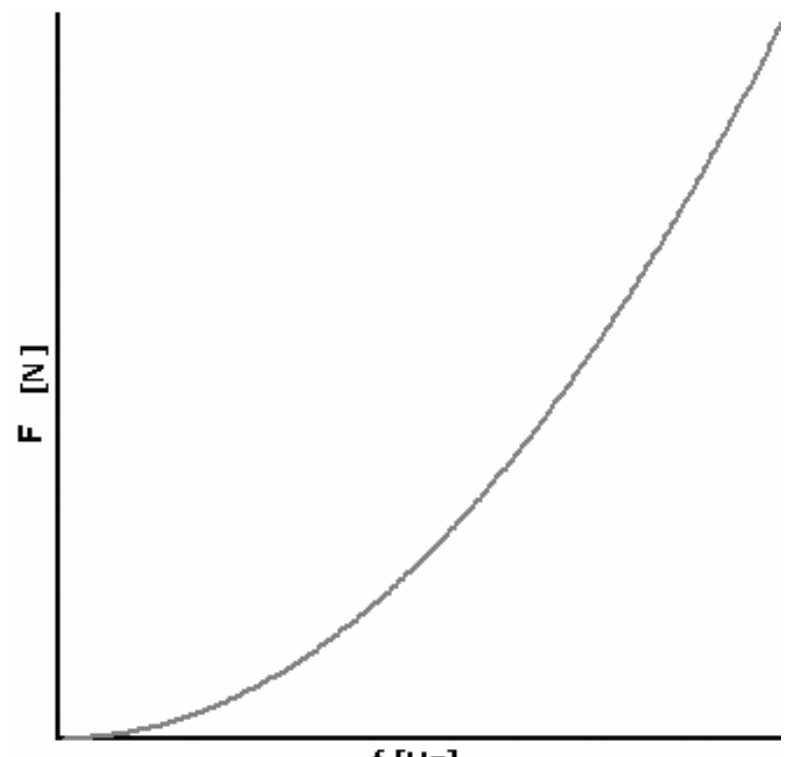

$\mathbf{f}[\mathrm{Hz}]$

Fig 11. The character of the loading of the flapping wing

As can be seen, a small change in the frequency results in quite a marked increase in the loading for higher frequencies. It is therefore reasonable to assume that this method will not allow the speed to be increased too much, taking into consideration that currently existing mechanisms are at material limits even for the pure hovering.

The second method of increasing airspeed would require the whole surface stroke to be shifted backwards. This method is quite difficult to implement mechanically and constrained by simple geometry. The wings cannot hit each other or any other aircraft components. The increase in airspeed increase is therefore also constrained in this case.

Because of these reasons the conclusion can be drawn that entomopters will not fly too fast. Therefore their application is also constrained despite projected high performance in hovering and slow flight.

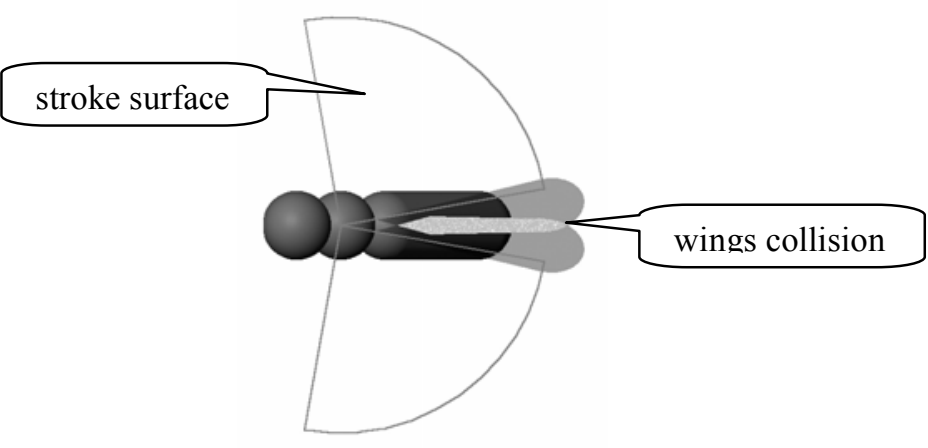

Fig 12. Shifting the whole stroke surface backwards

\section{Comparison of constraints on MAV configurations}

Based on the considerations presented it is possible to create the following table of MAV characteristics.

As can be seen, fixed wing MAVs are best FOR forward flight, entomopters are most suitable for hovering, and rotary MAVs are the most versatile. Mission types can therefore be assigned as follows:

$$
\begin{array}{ll}
\text { Fixed wings } & \begin{array}{l}
\text { - long range and/or long duration missions } \\
\text { in open space }
\end{array} \\
\text { Rotary } & \begin{array}{l}
\text { - short duration or long duration missions } \\
\text { with ,refuelling” in constrained space }
\end{array} \\
\text { Flapping wings } & \text { - indoor missions }
\end{array}
$$

\section{Examples of application}

\subsection{Outdoor NBC emergency reconnaissance}

In this type of mission operators will not be eager to stay close to the accident site. Any change in the direction of the wind could be risky, particularly if gaseous agents were released. Keeping a safe distance is required and thus promotes the fixed wing solution.

\subsection{Crowd control}

This is a police application. They have the need to observe crowd behaviour with as little interference as possible. They do not want to stay too close to the crowd to avoid making it angry. The same result would occur if a large flying object were flying over them. MAV would 
therefore be an ideal solution if the required range and flight endurance were available. Once again, fixed wing
MAV seems to be the best.

Table. MAV characteristics

\begin{tabular}{|c|c|c|c|c|c|c|}
\hline & \multicolumn{3}{|c|}{ Fast flight } & \multicolumn{3}{c|}{ Hover } \\
\hline & possibility & efficiency & manoeuvrability & possibility & efficiency & manoeuvrability \\
\hline $\begin{array}{c}\text { Fixed } \\
\text { wing }\end{array}$ & yes & very good & very good & no & - & - \\
\hline Rotary & yes & poor & moderate & yes & poor & moderate \\
\hline Flapping & no & - & - & yes & very good & very good \\
\hline
\end{tabular}

\subsection{Surveillance of suspect facilities}

The requirements are similar to the previous since the presence of the police forces and/or large flying objects would influence the behaviour of the suspects, thus frustrating the plans of the police. On the other hand, sufficient reconnaissance of the facility is required, particularly before any actions against organised criminals. In this case fixed wing MAV would also be advantageous.

\subsection{Regular pollution inspection}

The possibility to sample the industrial fumes released into the atmosphere would be quite advantageous in the view of global warming. In this case, neither long range nor long endurance is required. Inspectors need only to stop relatively close to a site and take the sample as fast as possible (not noticed by the management inspected company). On the other hand, the ability to negotiate fast winds would be required because of the need to fly to high chimney tops. This task should be performed by a rotary MAV.

\subsection{Road accident documentation}

In this case, police would not be interested in long endurance nor long range since they have to reach the site personally anyway and prepare the documentation as quickly as possible so as not to cause excessive traffic jams. On the other hand, a stable, hovering platform with VTOL capability would be advantageous. Rotary configuration would therefore be the best.

\subsection{Urban traffic management}

In this case MAVs would supplement existing stationary surveillance systems. The worst disadvantage of the stationary systems is that they provide only partial information. A large number of systems would however be required to provide complete information even for a moderate size city. The number of systems required could be significantly decreased if some of them were installed on MAVs. Problems could be located by stationary systems and solved with the help of mobile ones. After one problem is solved, mobile systems could fly to another site. Long range and long endurance would not be required because vehicles could be "refuelled" from the existing electrical urban infrastructure. Moreover, they would not be required to fly all the time, but only during relocation. In such a case, once again rotary vehicles would best serve the mission.

\subsection{Search for survivors}

The need for a system capable of finding survivors became apparent after the terrorist attack on the World Trade Centre. Rescuers could have saved more lives if they had a system designed to fly across the rubble, search for people, and deliver necessary food and medications before the rubble was removed. Instead they first had to remove debris, which took several weeks, and then they found no one alive. Flapping MAVs would be a perfect solution in this case. They could penetrate the rubble regardless of any obstacles that could stop other vehicles. This kind of system can however also be useful in many other cases such as earthquakes hurricanes, or mining disasters.

\subsection{Pipeline inspection}

From time to time there is the need to inspect various types of pipelines from inside. This task can be assigned to different types of robots, but only a flapping MAV will be able to perform such a mission without any preparation even inside very complicated pipeline system. Pipes can change the course many times in any direction. They only need to be large enough to accommodate the MAV

\subsection{High risk indoors inspection}

In this case flapping MAVs would provide more flexibility when inspecting dangerous laboratory or industrial facilities. Similar to example 3.6, this task can also be served by a fixed inspection system, but a greater number of systems would be necessary to provide the complete information. Instead, flapping MAV could take care of about everything regardless of any changes made to the installations. 


\section{Conclusion}

MAVs can serve in many different applications and fulfil requirements that sometimes excluding each other. Fortunately, different MAV configurations exhibit supplementary characteristics. It is therefore possible to tailor a certain requirement with a certain configuration:

- $\quad$ Fixed wing MAVs for long endurance outdoors missions

- Rotary MAVs for short endurance outdoors missions with hover

- $\quad$ Flapping MAVs for indoors missions

\section{Acknowledgements}

The rector of the Warsaw University of Technology through grant 503R11320264004 supported this work.

\section{References}

1. Dickinson M.H., Lehmann F.O., Sane S.P. Wing rotation and the aerodynamic basis of insect flight // Science, - 1999, 18 June. - Vol 284. -P. 1954-1960.

2. Dohring C. Der Schub des schlagenden Flügels und seine Anwendung zur Grenzschichtbeeinflussung - $\quad$ eine experimentelle und numerische Untersuchung: Doctor thesis at the Fakultät für Luft - und Raumfahrttechnik der Universität der Bundeswehr München. - 1998, May 28. - S. 25-30.

3. Fearing R.S., Chiang K., Dickinson M. et al. Wing transmission for a micromechanical flying insect // Proceedings of IEEE International Conference on Robotics and Automation, ICRA'2000. - 2000. - Vol 2. - P. 1509-1516.

4. Galinski C., Lawson N., Żbikowski R. Delta wing with leading edge extension and propeller propulsion for fixed wing MAV // Proceedings of $24^{\text {th }}$ International Congress of the Aeronautical Sciences, Yokohama, 2004. Yokohama, 2004. - Paper ICAS 2004-1.10.5.

5. Jones K.D., Platzer M.F. Experimental investigation of the aerodynamic characteristics of flapping-wing micro air vehicles $/ / 41^{\text {st }}$ Aerospace Sciences Meeting\&Exhibit, 6-9 January 2003, Reno, NV. - 2003. - Paper AIAA-2003-0418.

6. Rist U. Instability and Transition Mechanisms in laminar Separation Bubbles // Low Reynolds Number Aerodynamics on Aircraft Including Applications in Emerging UAV Technology. Von Kármán Institute Lecture Series RTO/AVT104. - Rhode-Saint-Genèse, 2003, 24-28 November. - Paper 5.

7. Rist U., Augustin K. Control of Laminar Separation bubbles"// Ibid. - Paper 6.
8. Sane S.P., Dickinson M.H. The aerodynamic effects of wing rotation and a revised quasisteady model of flapping flight // The Journal of Experimental Biology. - 2002. - Vol 205. - P. 1087-1096.

9. Van den Berg C., Ellington C.P. The threedimensional leading-edge vortex of a "hovering" model hawk moth // Philosophical Transactions of the Royal Society of London, Series B, Biological Sciences 352, 1351. - 1997. - P. 329-340.

10. Van den Berg C., Ellington C.P. The vortex wake of a "hovering" model hawk moth. Philosophical Transactions of the Royal Society of London, Series B. // Biological Sciences. 1997. - Vol 352, No 1351. - P. 317-328.

11. Watkins S., Melbourne W. Atmospheric Winds: Implications for MAVs"// Proceedings of the XVIII International UAV Conference, 31 March - 2 April 2003, Bristol, UK. - Bristol, 2003. - Paper 26.

12. Website:http://news.bbc.co.uk/1/hi/technology/3 579232.stm

13. Website:http://robots.engadget.com/entry/72785 20217452629/

14. Website:http://www.space.com/businesstechnol ogy/flying_robot_040819.html

15. Wojciechowski ${ }^{-}$. Active Control of the Boundary Layer on the Laminar Airfoil // Inženýrská Mechanika'99. - Svratka, 1999 1720 Květen. - P. 813-818.

16. Żbikowski R. Cycle of a wing beat // Unmanned Vehicles. - 2003 July-August. -Vol 8, No 4. - P. 35-38.

17. Żbikowski R., Galiński C., Pedersen C.B. A four-bar linkage mechanism for insect-like flapping wings in hover: Concept and an outline of its realisation // Journal of Mechanical Design /Transactions of the ASME. - 2005 July. - Vol 127. - P. 817-824.

18. Żbikowski R., Pedersen. C.B., Ansari S.A. et al. Aeromechanical Aspects of Insect-Like Flapping MAVs // Low Reynolds Number Aerodynamics on Aircraft Including Applications in Emerging UAV Technology. Von Kármán Institute Lecture Series RTO/AVT104. - Rhode-Saint-Genèse, 2003， 24-28 November. - Paper 10. 